\title{
Helina
}

\section{Pan African Health Informatics Association (HELINA)}

\author{
Prepared by Dr. Ghislain B. Kouematchoua Tchuitcheu
}

\section{HELINA 2018 Conference}

The 2018 Pan African Health Informatics in Africa conference took place from 3rd to 5th December 2018 in Nairobi, Kenya. The conference was co-hosted by the Ministry of Health of Kenya and the Kenya Health Informatics Association (KeHIA), co-chaired by Dr. Ghislain Kouematchoua (HELINA President) and Dr. Tom Olouch (KeHIA Chairman) and sponsored by RTI International, IBMI Moi University, Malteser International, and IntelliSOFT Consulting Kenya. KeHIA and Kenya Ministry of Health co-hosted the conference back-to-back with the OpenMRS Implementers Meeting.

The conference focused on the use of technology to strengthen health systems in the African Region. Issues of specific interest were the development and implementation of integrated e-Health plans and policies that enable capacity building for eHealth professionals, improved quality of health information, and promotion of the meaningful use of health data to support and grounded decision-making. Further important topics were enabling access to essential medical supplies through improved supply chain and logistics, development of sustainable health information systems for service delivery, and innovative health financing models that improve access to health. Another highlight was the role of digital health in health surveillance systems particularly due to emerging health threats from non-communicable diseases (NCDs) and the core participatory role of the client in detection, response, treatment, and care. Special attention was paid to the role of e-Health in achieving the Sustainable Development Goals (SDG) passed by the UN in September 2015 and specifically to goal 9, target 9c which aims to "Significantly increase access to information and communications technology and strive to provide universal and affordable access to the Internet in the least developed countries by $2020^{4}$.

\section{Scientific Program}

The scientific program committee was chaired by Prof. Nicky Mostert from South Africa, and co-chaired by Prof. Georges Nguefack from Cameroon, Dr. Chris Olola from Kenya, and Dr. Frances da-Costa Vroom from Ghana. The SPC received after the call for papers a total of 76 submissions out of which 12 full research papers (16\%), 6 workin-progress papers ( $8 \%$ ), and 33 case studies and experience or concept papers $(43 \%)$ were accepted. Twenty-five (33\%) papers were rejected or retracted. A double-blind peer review process was used for evaluating each paper. All submissions were anonymized before being submitted to at least 2 reviewers based on their expertise. The SPC decision was based on the recommendations and comments from reviewers. Accepted full research papers were published in a special edition of the Journal of Health Informatics in Africa (JHIA) - http://www.jhia-online. org - and the accepted work-in-progress papers, case studies/experience papers were electronically published in the conference Proceedings with ISBN by Koegni-eHealth and are available on the conference website - http://conf.helina-online.org.

Presentations at the conference indicated that a lot of work is being done towards harnessing the potential of technology systems to build sustainable health systems in Africa.

\section{Participation}

The Conference attracted 163 participants from 26 countries - Cameroon, Canada, Democratic Republic of Congo, Ethiopia, France, Ghana, Germany, Haiti, Ireland, Kenya, Lesotho, Liberia, Malawi, Mozambique, Nigeria, Rwanda, Sierra Leone, South Africa, Sweden, Switzerland, Tanzania, Uganda, United Kingdom, and United States of America. They were primarily members of academia, researchers, and health informatics practitioners.

Delegates also provided feedback on areas of improvement for future conferences. Suggestions included addition of more keynote talks, earlier access to the program, increased time/session for networking and peer collaborations, more marketing or preevents to increase awareness of HELINA and KeHIA in the industries, etc.

\section{Sponsorphip}

HELINA 2018 had four commitments from sponsors RTI international, IBMI Moi University, Malteser International, and IntelliSOFT Consulting Limited.

\section{AAcknowledgements}

The author would like to thank the HELINA SPC chair and the Local organizing committee for their contributions to this report.

\section{Regional Editor}

Ghislain B. Kouematchoua Tchuitcheu, PhD, FIAHSI

IMIA Vice President for HELINA

E-mail: kouematchoua@helina-online.org ghislain.k@koegni-ehealth.org

www.helina-online.org 